\title{
Spontaneous Internal Carotid Artery Occlusion and Rapid Cerebral Aneurysm Progression: Case Series and Literature Review
}

\author{
Bakhsh Saaquib, MD', Toll Valerie, MD', Neimann David, MD², Michael Chen, MD'
}

Purpose: An accurate determination of the natural history of a cerebral aneurysm has implications on management. Few risk factors other than female gender and cigarette smoking have been identified to be associated with cerebral aneurysm progression, particularly rapid progression.

Materials and Methods: This case series and literature review serves to illustrate a relationship between spontaneous carotid occlusion and rapid enlargement of cerebral aneurysms.

Results: In our case series, we demonstrated that increased hemodynamic stress on collateral vessels caused by a spontaneous carotid occlusion may contribute to unusually rapid aneurysm growth and/or rupture.

Conclusion: Spontaneous carotid occlusive disease may be considered a risk factor for rapid cerebral aneurysm progression and/or rupture that may warrant more aggressive management options, including more frequent surveillance imaging in previously treated aneurysms.

Key Words : Aneurysm; Carotid occlusion; Rapid progression; Risk factors

Aneurysmal subarachnoid hemorrhage is not only one of the most common causes for stroke among young adults [1], but also usually leads to disabling neurologic deficits or death [2]. The majority of the cited risk factors associated with cerebral aneurysm

'Departments of Neurological Surgery and Neurological Sciences, Rush University Medical Center, Chicago, IL, USA

${ }^{2}$ Department of Neurological Surgery, University of Wisconsin School of Medicine and Public Health, Madison, WI, USA

Received June 4, 2014; accepted after revision June 30, 2014.

Correspondence to: Michael Chen, MD, Departments of Neurological Surgery and Neurological Sciences, Rush University Medical Center, 1725 West Harrison Street Suite 855, Chicago, IL 60612, USA

Tel. 1.312.563.2817 Fax. 1.312.942.2176

E-mail: Michael_Chen@rush.edu

This is an Open Access article distributed under the terms of the Creative Commons Attribution Non-Commercial License (http://creativecommons.org/licenses/by-nc/3.0) which permits unrestricted non-commercial use, distribution, and reproduction in any medium, provided the original work is properly cited. relate to rupture [3-5]. A handful of risk factors have only recently been reported that relate to growth, likely as a result of the increased use and availability of noninvasive brain vascular imaging [2]. Of these, female gender and cigarette smoking have been cited as risk factors for cerebral aneurysm progression.

As such, surveillance imaging, blood pressure control and smoking cessation currently make up the essence of any long-term management plan for patients diagnosed with a cerebral aneurysm, regardless of prior surgical treatment. Determining the frequency of surveillance imaging is arbitrary and often spans up to twelve months between studies.

In this case series and literature review, we highlight a possible relationship between carotid occlusive disease and rapid cerebral aneurysm progression. The implication of these findings is that in patients with a previously diagnosed or treated cerebral aneurysm, this non-modifiable anatomic finding may either justify 

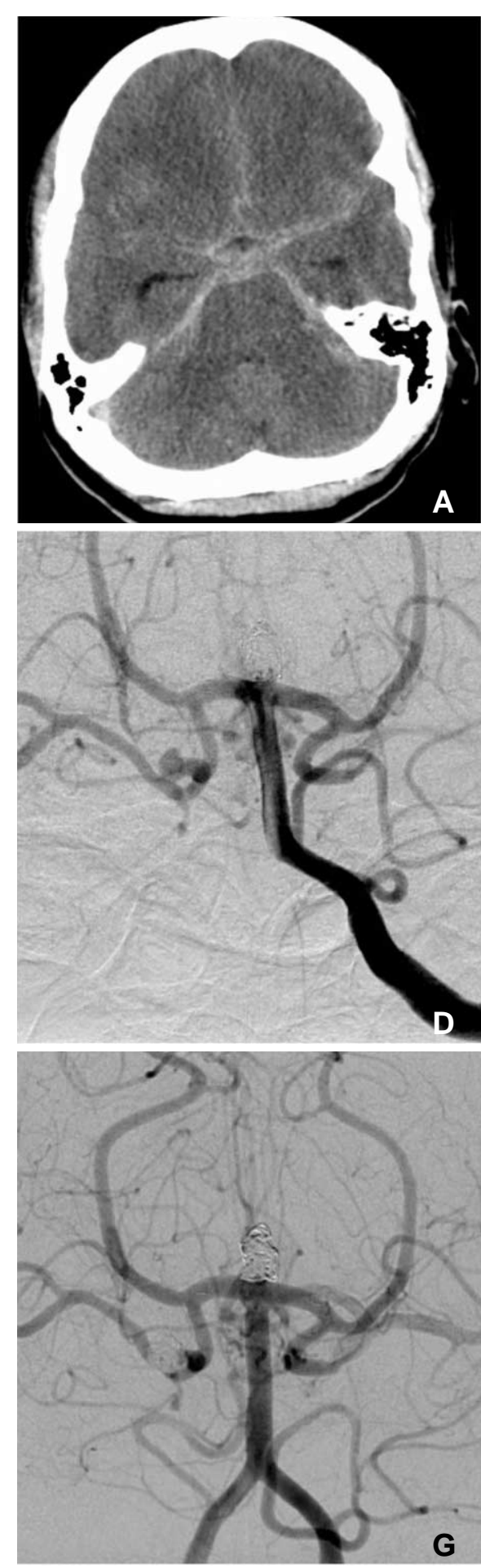
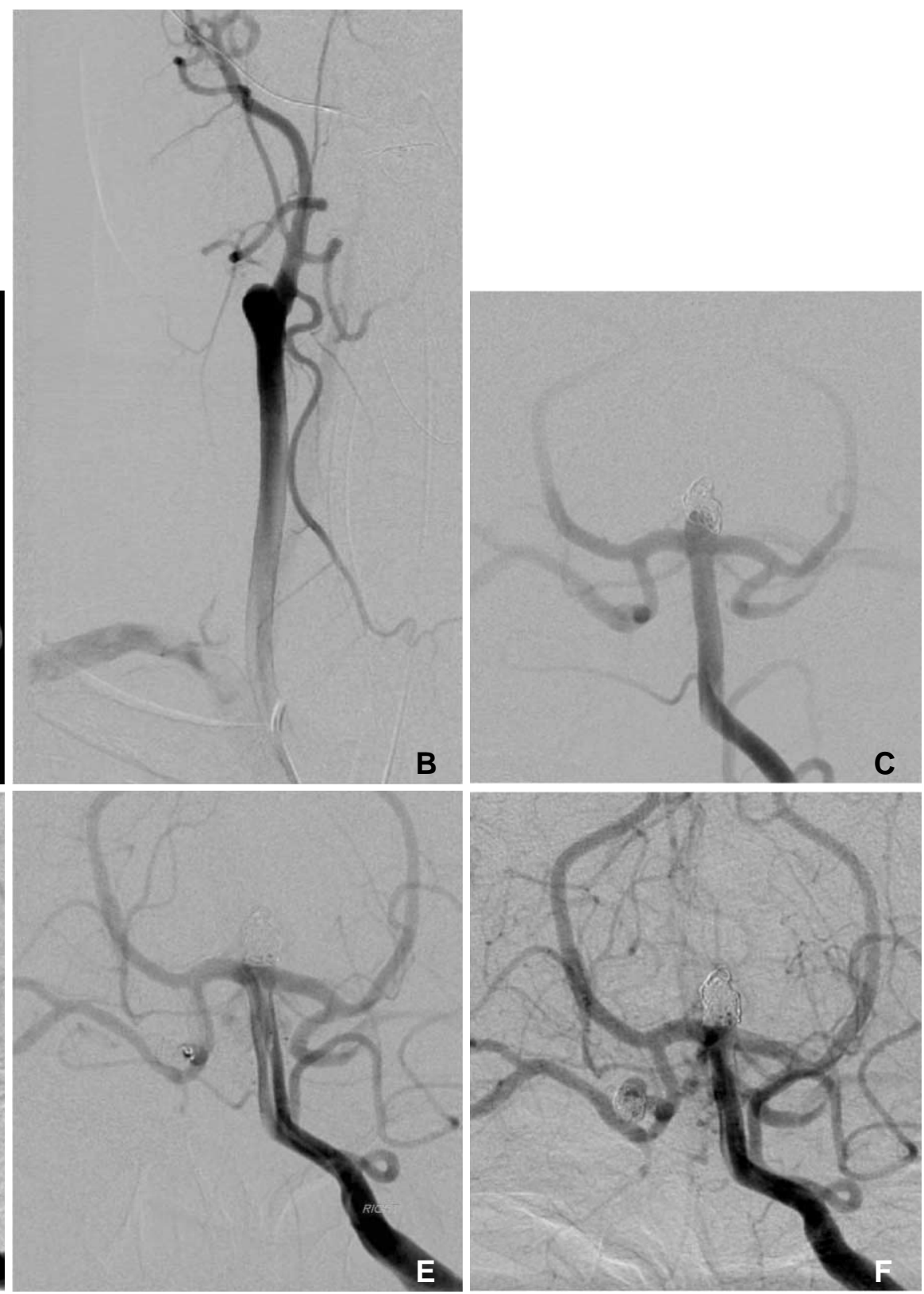

Fig. 1. A. Noncontrast head CT demonstrating diffuse basilar cistern and right Sylvian fissure subarachnoid hemorrhage. B. AP right common carotid angiography demonstrating a carotid occlusion at the bulb. C. AP left vertebral angiography showing neck recanalization of the previously embolized basilar terminus aneurysm and a $2 \mathrm{~mm}$ laterally directed oblong right posterior communicating artery aneurysm. D. AP left vertebral angiography demonstrating enlargement of the right posterior communicating artery aneurysm with a significant change in morphology. E. AP left vertebral angiography demonstrating Raymond 2 occlusion of the right posterior communicating artery aneurysm with adequate dome occlusion. F. AP left vertebral artery angiography demonstrating aneurysm dome growth. G. AP left vertebral artery angiography demonstrating durable occlusion of both the basilar terminus aneurysm and right posterior communicating artery aneurysm. 
anatomic exclusion, or at the very least, warrant more frequent surveillance of brain vascular imaging.

\section{Case One}

A 48-year-old woman with a history of cocaine use was found unconscious by neighbors and brought to the hospital where she was found to be drowsy, but with an otherwise normal neurologic examination. A plain head CT scan demonstrated diffuse basilar cistern subarachnoid hemorrhage (Fig. 1A). A basilar terminus aneurysm was embolized and a small right $2 \mathrm{~mm}$ posterior communicating artery aneurysm was seen in conjunction with a right cervical internal carotid artery (ICA) occlusion (Fig. 1B).

Four months later, she returned with a second episode of subarachnoid hemorrhage and there appeared to be significant neck recanalization of her previously embolized basilar terminus aneurysm and an unchanged appearance of the laterally directed rightsided $2 \mathrm{~mm}$ posterior communicating artery aneurysm (Fig. 1C). The basilar terminus aneurysm remnant was embolized with the adjunctive use of a horizontally placed intracranial stent from one P1 segment to the other P1 segment. She had a favorable angiographic and clinical result.

Two weeks later, she developed a right oculomotor nerve palsy and evaluation showed no acute hemorrhage but remarkable growth in size and morphology of her right posterior communicating artery aneurysm, now nearly $5 \mathrm{~mm}$ (Fig. 1D). The previously seen oblong shape now became multilobulated. This aneurysm was embolized to satisfactory occlusion (Fig. 1E).

Two weeks later, surveillance catheter angiography demonstrated what appeared to be further growth of the aneurysm dome, and not simply coil compaction (Fig. $1 \mathrm{~F})$. This was embolized to a satisfactory occlusion. Subsequent surveillance of catheter cerebral angiographic images at 6 months and one year have shown durable complete occlusion (Fig. 1G).

\section{Case Two}

A 55-year-old woman developed right carotid occlusive disease after subtotal resection and radiation of a right optic nerve glioma nearly 12 years prior. She was found to have an anterior communicating artery aneurysm that was clipped. Four months prior to presentation, she was found to have a $4 \mathrm{~mm}$ inferiorly directed anterior communicating artery aneurysm residual, (Fig. 2A) which was embolized with coils with a final result intentionally leaving some neck filling to minimize the risk of impairing flow to the contralateral hemisphere (Fig. 2B).

Four months later, she developed a severe headache and was found to have subarachnoid hemorrhage in the basilar cisterns and interhemispheric fissure (Fig. 2C). Catheter angiography demonstrated the previously visualized embolized anterior communicating artery aneurysm to have increased in size to $15 \mathrm{~mm}$ in maximal diameter (Fig. 2D). Fortunately, embolization of the aneurysm dome proceeded uneventfully (Fig. $2 \mathrm{E}$ ) and she was discharged home with a favorable recovery and normal neurologic examination.

\section{DISCUSSION}

Hemodynamic stress is a well-known physiologic risk factor for cerebral aneurysm pathogenesis [6-10]. This has been further described as a sequential, repetitive reversal of flow within the dome of the aneurysm. Blood enters the cavity along the proximal wall and then emerges distally during systole. During diastole, the flow direction is reversed; these rapid changes in direction of blood flow continually stress the intima and neck of the cavity and may contribute to aneurysm formation and progression [11]. Histologically, a thinning of the tunica media can be observed, the critical component of the arterial wall that is responsible for compliance [12].

Cervical carotid segment stenosis and/or occlusion, a relatively common finding [13] in about $3 \%$ of the general population, can increase this type of hemodynamic stress within the remaining collateral vessels. In the case of an ICA occlusion, cerebral perfusion pressure drops as regional blood flow is maintained via autoregulated vasodilation and the rerouting of blood through compensatory pathways. In a small series presented by van Everdingen et al. patients with symptomatic unilateral ICA occlusion had increased contralateral flow in the internal carotid and basilar artery. This was quantified as a $130-170 \%$ increase in blood flow through the compensatory carotid artery [14].

A relationship between therapeutic carotid occlusion and cerebral aneurysm formation has been previously described. A literature review from 1970 - 2008 which reviewed data from 187 patients with therapeutic carotid artery occlusions, demonstrated a $4.3 \%$ rate of de novo aneurysm formation at an average duration of 9.1 years after the occlusion $[15,16]$; a related review also found a $0-4 \%$ frequency of de novo aneurysm formation in carotid ligation patients, with a majority of patients (10 out of 12) experiencing a hemorrhage [17]. 

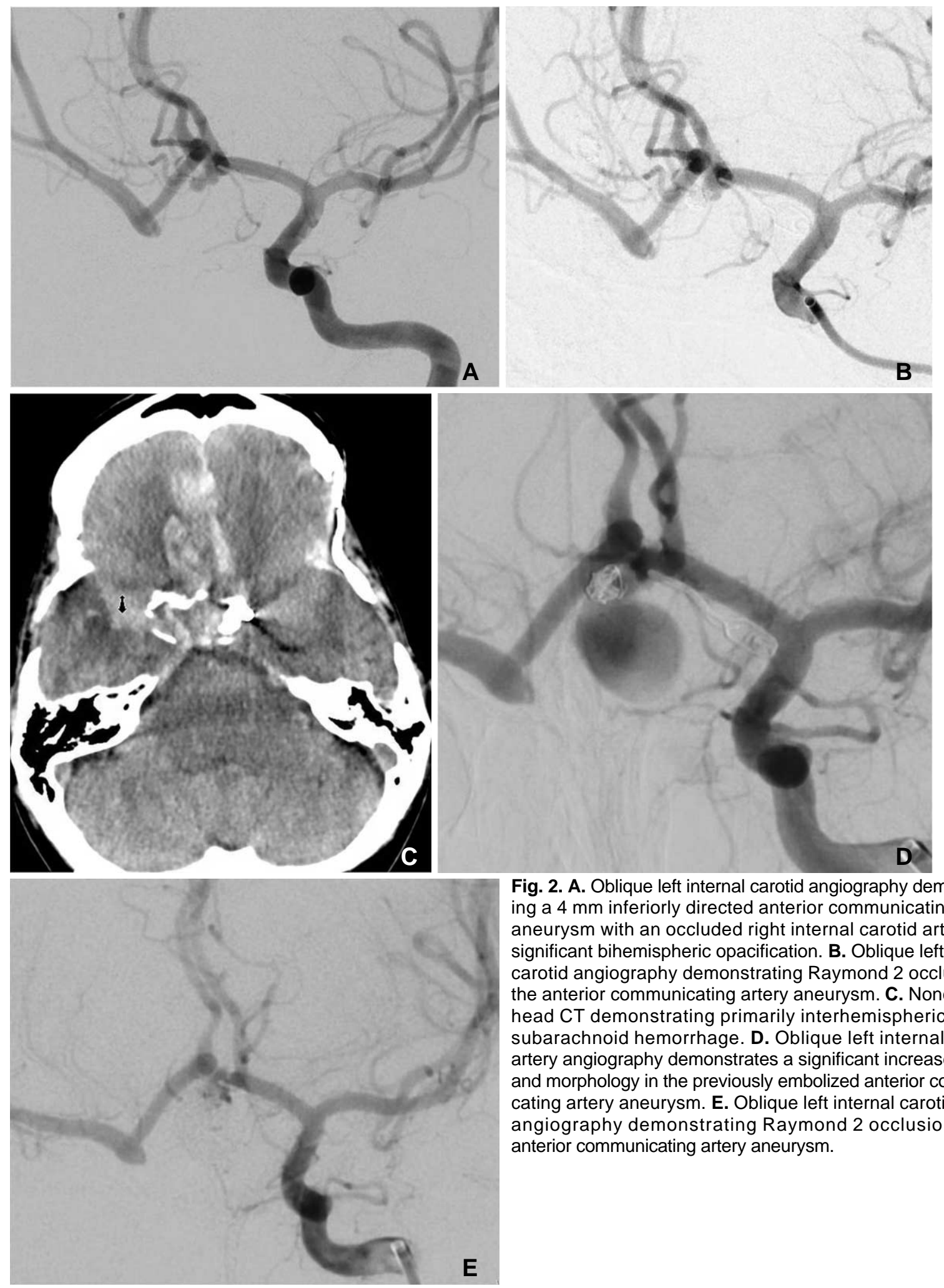

Fig. 2. A. Oblique left internal carotid angiography demonstrating a $4 \mathrm{~mm}$ inferiorly directed anterior communicating artery aneurysm with an occluded right internal carotid artery and significant bihemispheric opacification. B. Oblique left internal carotid angiography demonstrating Raymond 2 occlusion of the anterior communicating artery aneurysm. C. Noncontrast head CT demonstrating primarily interhemispheric fissure subarachnoid hemorrhage. D. Oblique left internal carotid artery angiography demonstrates a significant increase in size and morphology in the previously embolized anterior communicating artery aneurysm. E. Oblique left internal carotid artery angiography demonstrating Raymond 2 occlusion of the anterior communicating artery aneurysm.

Few reports exist, however, for an association with spontaneous carotid occlusions. In 1968, Jaffe et al. described a 79-year-old woman presenting with a right posterior communicating artery aneurysm ten years after a stroke due to spontaneous occlusion of her left internal carotid [18].

In each of our cases (two weeks in case one and four months in case two), we witnessed an unusually rapid 
expansion of the aneurysm dome, despite what we thought was adequate occlusion with coil embolization. The most plausible mechanism appeared to be associated carotid occlusive disease. Incomplete embolization of the aneurysm could be regarded as an alternate explanation [19]. The extent of coiling varies in any case at the discretion of the surgeon and the conditions of the surgery, which determine the degree of satisfactory aneurysm occlusion. However, incomplete embolization usually leads to coil compaction but not necessarily actual growth of the aneurysm dome, as we witnessed in our series.

Rapid cerebral aneurysm progression has been scarcely reported in the literature. Of these include mycotic aneurysms associated with fungal infections in immunocompromised patients [20]. The primary mechanism of progression is described as invasive arteritis and subsequent elastic tissue digestion [21]. In these situations, hemodynamic stress likely plays less of a role. Arterial wall integrity is more severely compromised. In both of our cases, there was no evidence of an infection, yet rapid progression of the aneurysms was seen.

In summary, patients with carotid occlusive disease may be at risk for rapid aneurysm progression within the remaining patent cerebral vessels. The presumed mechanism is increased compensatory flow that exerts deleterious hemodynamic stress on the component of the arterial wall responsible for compliance, thereby contributing to aneurysm formation. Therefore, in these patients, there may be good reason to consider anatomic exclusion, while aggressively controlling blood pressure and possibly obtaining more frequent brain vascular surveillance imaging. Further development of quantitative flow models that look at this relationship between aneurysms and carotid occlusive disease may provide useful additional insight.

\section{References}

1. Sarti C, Tuomilehto J, Salomaa V, Sivenius J, Kaarsalo E, Narva EV, et al. Epidemiology of subarachnoid hemorrhage in finland from 1983 to 1985. Stroke 1991;22:848-853

2. Juvela S, Poussa K and Porras M, Factors affecting formation and growth of intracranial aneurysms: a long-term follow-up study. Stroke 2001;32:485-491

3. Ostergaard JR, Risk factors in intracranial saccular aneurysms. Aspects on the formation and rupture of aneurysms, and development of cerebral vasospasm. Acta Neurol Scand 1989;80:81-98

4. Longstreth WT Jr, Nelson LM, Koepsell TD, van Belle G. Cigarette smoking, alcohol use, and subarachnoid hemorrhage. Stroke 1992;23:1242-1249

5. Juvela S, Hillbom M, Numminen H, Koskinen P. Cigarette smoking and alcohol consumption as risk factors for aneurysmal subarachnoid hemorrhage. Stroke 1993;24:639-646

6. Alnaes MS, Isaksen J, Mardal KA, Romner B, Morgan MK, Ingebrigtsen $\mathrm{T}$. Computation of hemodynamics in the circle of willis. Stroke 2007;38:2500-2505

7. Castro MA, Putman CM, Sheridan MJ, Cebral JR. Hemodynamic patterns of anterior communicating artery aneurysms: a possible association with rupture. AJNR Am J Neuroradiol 2009;30:297302

8. Eldawoody H, Shimizu H, Kimura N, Saito A, Nakayama T, Takahashi A, et al. Simplified experimental cerebral aneurysm model in rats: comprehensive evaluation of induced aneurysms and arterial changes in the circle of Willis. Brain Res 2009; 1300:159-168

9. Meng H, Wang Z, Hoi Y, Gao L, Metaxa E, Swartz DD, et al. Complex hemodynamics at the apex of an arterial bifurcation induces vascular remodeling resembling cerebral aneurysm initiation. Stroke 2007;38:1924-1931

10. Chatziprodromou I, Tricoli A, Poulikakos D and Ventikos Y. Haemodynamics and wall remodelling of a growing cerebral aneurysm: a computational model. J Biomech 2007;40:412-426

11. Gonzalez CF, Cho YI, Ortega HV, Moret J. Intracranial aneurysms: flow analysis of their origin and progression. AJNR Am J Neuroradiol 1992;13:181-188

12. Brisman JL, Song JK, Newell DW. Cerebral aneurysms. N Engl J Med 2006;355:928-939

13. de Weerd M, Greving JP, Hedblad B, Lorenz MW, Mathiesen EB, O'Leary DH, et al. Prevalence of asymptomatic carotid artery stenosis in the general population: an individual participant data meta-analysis. Stroke 2010;41:1294-1297

14. van Everdingen KJ, Klijn CJ, Kappelle LJ, Mali WP, van der Grond J. MRA flow quantification in patients with a symptomatic internal carotid artery occlusion. The Dutch EC-IC By pass study Group. Stroke 1997;28:1595-1600

15. Arambepola PK, McEvoy SD, Bulsara KR, De novo aneurysm formation after carotid artery occlusion for cerebral aneurysms. Skull Base 2010;20:405-408

16. Timperman PE, Tomsick TA, Tew JM Jr., van Loveren HR. Aneurysm formation after carotid occlusion. AJNR Am J Neuroradiol 1995;16:329-331

17. Dyste GN, Beck DW. De novo aneurysm formation following carotid ligation: case report and review of the literature. Neurosurgery 1989;24:88-92

18. Jaffe ME, McHenry LC, Jr. Cerebral aneurysm following spontaneous carotid occlusion. Neurology 1968;18:1012-1014

19. Klein O, Colnat-Coulbois S, Civit T, Auque J, Bracard S, Pinelli C, et al. Aneurysm clipping after endovascular treatment with coils: a report of 13 cases. Neurosurg Rev 2008;31:403411

20. Gupta K, Radotra BD, Suri D, Sharma K, Saxena AK and Singhi P. Mycotic aneurysm and subarachnoid hemorrhage following tubercular meningitis in an infant with congenital tuberculosis and cytomegalovirus disease. Journal of Child Neurology 2012;27:1320-1325

21. Hurst RW, Judkins A, Bolger W, Chu A, Loevner LA. Mycotic aneurysm and cerebral infarction resulting from fungal sinusitis: imaging and pathologic correlation. AJNR Am J Neuroradiol 2001;22:858-863 